Copyright ( 2008 IEEE. Reprinted from IEEE Transactions on Power Delivery, 2007; 22 (2):765-771

This material is posted here with permission of the IEEE. Such permission of the IEEE does not in any way imply IEEE endorsement of any of the University of Adelaide's products or services. Internal or personal use of this material is permitted. However, permission to reprint/republish this material for advertising or promotional purposes or for creating new collective works for resale or redistribution must be obtained from the IEEE by writing to pubs-permissions@ieee.org.

By choosing to view this document, you agree to all provisions of the copyright laws protecting it. 


\title{
An Adaptive Differentiation Filter for Tracking Instantaneous Frequency in Power Systems
}

\author{
Rastko Živanović, Member, IEEE
}

\begin{abstract}
This paper presents an application of adaptive differentiation filter in tracking instantaneous frequency in electrical power systems. For each new sample, the filter automatically selects an optimal window length that maximizes measurement accuracy. Large window length is selected if the frequency is slow varying or steady state, to increase efficiency in filtering noise and harmonics. For fast-varying frequency, the window length is automatically reduced in order to make frequency tracking more accurate, sacrificing filtering efficiency. Automatic selection of the optimal window length that balances between tracking and filtering performance is the unique feature of this technique. This paper concludes with the presentation of the representative results obtained in the simulation study as well as in some practical applications. The results show the adaptive differentiation filter gives accurate frequency measurement under both steady-state and dynamic conditions.
\end{abstract}

Index Terms-Digital signal processing, frequency measurement, frequency relaying, power system frequency.

\section{INTRODUCTION}

$\mathbf{T}$ HE most common approach in designing the frequency measurement algorithm is based on projecting the voltage signal on some set of orthogonal basis functions. If the basis functions are trigonometric functions, the outcome is the discrete Fourier transform (DFT) method [1]. The basis functions are designed for the fundamental frequency and harmonics of that frequency which makes the method sensitive to large frequency variations. The least-squares technique can also be used for measuring frequency [1]. In this approach, the voltage signal is represented with a sinusoidal function. Since the frequency is a nonlinear parameter, this model needs to be linearized by applying Taylor's series expansion. Frequency is calculated using the least-squares algorithm on a fixed window length. A problem with this approach is that the linearization is valid only in a narrow range around the expected frequency. The method is inaccurate for large frequency excursions.

The first step in the demodulation-based frequency measurement technique [2], [3] is a transformation that represents voltage signals recorded in all three phases with two orthogonal components - real and imaginary parts of the complex signal. In the next step, demodulation removes the nominal frequency from the complex signal. Deviation from the nominal frequency can be estimated from the instantaneous phase angle of such

Manuscript received September 2004; revised July 2005 and August 2006. This work was supported in part by the National Research Foundation of South Africa and in part by the ESKOM Tertiary Education Support Program. Paper no. TPWRD-00465-2004.

The author is with the University of Adelaide, Adelaide SA 5005 Australia (e-mail: rastko.zivanovic@ adelaide.edu.au). Paper no. TPWRD-00465-2004.

Digital Object Identifier 10.1109/TPWRD.2007.893368 a demodulated complex signal using the differentiation filter [4]. Another approach is to use a simple backward difference followed by a low-pass filter [3]. Since the filter parameters and window length are fixed, these techniques are not optimal for tracking the time-varying frequency.

In this paper, we present a novel adaptive differentiation filter to be used as a last step of the demodulation-based frequency measurement algorithm. By continuously adapting the window length according to the fundamental frequency change, this algorithm optimizes the performance in a sense of keeping balance between accurate tracking and efficient filtering of noise and nonfundamental components. This paper is divided as follows: in Section II, the differentiation filter for frequency estimation is derived, Section III discusses the filter accuracy as a function of window length, and Section IV presents the algorithm for selecting the optimal window length. The results obtained in testing the algorithm, which are presented in Sections V and VI of this paper, confirm good measurement accuracy in the presence of abrupt changes during switching conditions, high noise levels, and nonfundamental frequencies.

\section{FREQUENCY ESTIMATION}

\section{A. Instantaneous Voltage Phase Angle}

Samples $(q=1,2, \ldots)$ of three-phase voltages $\left(v_{a}(q)\right.$, $\left.v_{b}(q), v_{c}(q)\right)$ are used to estimate the instantaneous phase angle. First, the orthogonal components $v_{d}(q)$ and $v_{q}(q)$ of a complex voltage signal are obtained through the orthogonal Clarke transformation [5]

$$
\left[\begin{array}{l}
v_{d}(q) \\
v_{q}(q)
\end{array}\right]=\sqrt{\frac{2}{3}}\left[\begin{array}{ccc}
1 & -\frac{1}{2} & -\frac{1}{2} \\
0 & \frac{\sqrt{3}}{2} & -\frac{\sqrt{3}}{2}
\end{array}\right]\left[\begin{array}{l}
v_{a}(q) \\
v_{b}(q) \\
v_{c}(q)
\end{array}\right] .
$$

Second, the complex voltage signal $v_{d}(q)+j v_{q}(q)$ is shifted down (demodulated) in frequency domain to the angle value that corresponds to the nominal fundamental frequency $f_{0}=\omega_{0} / 2 \pi$. Finally, the instantaneous phase angle is calculated using [2] and [3]

$$
\varphi_{m}(q)=\arg \left(\left(v_{d}(q)+j v_{q}(q)\right) e^{-j q \theta_{0}}\right)
$$

where $\theta_{0}=\omega_{0} T_{s}$ is the sampling angle, and $T_{s}$ is the sampling interval.

It should be noted that under nominal frequency conditions, the phase angle (2) is constant. When the frequency deviates from nominal, the angle (2) is a slow time-varying nonoscillatory signal. Oscillatory components appear in the instantaneous phase angle due to additional harmonic components and unbalance condition in the three-phase voltage signals. 


\section{B. Differentiation Filter}

The problem with the commonly used two-sample frequency estimation technique [3]

$$
\hat{f}(q)=f_{0}+\frac{\varphi_{m}(q)-\varphi_{m}(q-1)}{2 \pi T_{s}}
$$

is the high-sensitivity-to-noise and oscillatory components in $\varphi_{m}$. The approach that is more general and overcomes this problem would be to approximate the phase-angle signal with a polynomial model and then take the first-order coefficient as a frequency estimate. We found that the linear model is sufficient in this application. Such approximation is applied locally, on a left-sided data window of length $n+1$, and it is obtained through fitting samples of the signal $\varphi_{m}$ to a linear model by minimizing the least-squares objective function

$$
\frac{1}{n+1} \sum_{k=0}^{n}\left[\varphi_{m}(q-k)-\varphi(q)+\Delta \omega(q) k T_{s}\right]^{2}
$$

where the parameters of the local linear model for the sample $q$ are $\varphi(q)$ and $\Delta \omega(q)$. It is possible to solve the least-squares problem (4) in the closed form. The closed-form solution of the parameter $\Delta \omega(q)$ for the window length $n+1$ is obtained in the nonrecursive differentiation filter form

$$
\Delta \hat{\omega}(q, n)=-\frac{1}{T_{s}} \sum_{k=0}^{n} h(k, n) \varphi_{m}(q-k)
$$

where the weight sequence $h(k, n)$ (impulse response of the filter) is calculated using the following formula:

$$
h(k, n)=\frac{6(2 k-n)}{n(n+1)(n+2)} .
$$

Hence, the instantaneous frequency estimator at the sample $q$ is given by

$$
\hat{f}(q, n)=f_{0}+\frac{\Delta \hat{\omega}(q, n)}{2 \pi} .
$$

If the window length is comprised of two samples $(n=1)$, the differentiation filter (5) and (7) is reduced to (3).

\section{ACCURACY AS THE FUnCTION OF Window LENGTH}

\section{A. Performance Parameters}

The window length of the filter (5) should adapt to the change in instantaneous phase angle $\varphi_{m}$, the long window for steadystate or slow-varying angle, and the short window for the fastvarying angle. The long data window will efficiently filter oscillatory components and noise, while the short window will reduce bias in tracking the phase-angle (frequency) variations. The optimal window length should cause a balance between filtering and tracking performance [6]. To find such an optimal solution, it is necessary to establish expressions for bias and variance estimation as a function of window length. These estimators are derived in the Appendix and presented here

$$
\begin{aligned}
\hat{b}_{\Delta \omega}(q, n) & =-\frac{n}{2 f_{s}} \Delta \omega^{\prime}(q) \\
\hat{\sigma}_{\Delta \omega}(n) & =\sqrt{\frac{12}{n(n+1)(n+2)}} f_{s} \sigma_{\varepsilon}
\end{aligned}
$$

where $f_{s}=1 / T_{s}$ is the sampling frequency and $\Delta \omega^{\prime}(q)$ is the first derivative of $\Delta \omega(q)$. The standard deviation $\sigma_{\varepsilon}$ in (9) of the angle $\varphi_{m}$ measurement error $\varepsilon$ is estimated in the robust way using the median absolute deviation (MAD) estimator [7]

$$
\hat{\sigma}_{\varepsilon}=\frac{\operatorname{median}\left(\left|\Delta \varphi_{m}\right|\right)}{0.6745 \sqrt{2}}
$$

where each entry of the vector $\Delta \varphi_{m}$ is calculated as the difference between two consecutive samples of $\varphi_{m}$. In the estimators (8)-(10), we assumed that the measurement error $\varepsilon$ belongs to the class of independently and identically distributed random numbers with zero mean and variance $\sigma_{\varepsilon}^{2}$.

The bias (8) is a deterministic error defined by the rate of the change of frequency $\Delta \omega^{\prime}(q)$ at the point $q T_{\mathrm{s}}$, window length, and sampling frequency. The sampling frequency should be constant which makes practical implementation easier, while the window length will vary to control bias in frequency tracking. For $\Delta \omega^{\prime}(q) \neq 0$, an increase of $n$ results in the bias increase. The standard deviation (9) represents the stochastic error determined by the standard deviation $\sigma_{\varepsilon}$, window length, and sampling frequency. According to (9), for a constant sampling frequency, the varying window length controls the standard deviation of the frequency estimate: the factor $\sqrt{12 /(n(n+1)(n+2))}$ in (9) decreases with the increase of $n$. By increasing the window length, we reduce the estimator (5) variance (i.e., the filtering efficiency is improved).

Mean square error (MSE) criterion gives an estimate of local accuracy of the filter (5) for a specified window length. This local MSE criterion combines both bias and standard deviation in the following expression [8]:

$$
\hat{J}_{\Delta \omega}(q, n)=\hat{b}_{\Delta \omega}^{2}(q, n)+\hat{\sigma}_{\Delta \omega}^{2}(n) .
$$

The straightforward way of determining the optimal window length by solving $\partial \hat{J}_{\Delta \omega}(q, n) / \partial n=0$ is not possible in practice because the rate of change $\Delta \omega^{\prime}(q)$ in (8) is not known in advance. However, we can use this criterion to determine the minimum window length to be used for tracking the maximal expected frequency change with the best possible filtering efficiency.

For example, if the sampling frequency $f_{\mathrm{S}}=640 \mathrm{~Hz}$, maximal rate of frequency change $f^{\prime}=40 \mathrm{~Hz} / \mathrm{s}$, and standard deviation of the phase-angle measurement error $\sigma_{\varepsilon}=0.6 \%$ are specified, then the minimum window length that corresponds to the minimum of the MSE criterion (11) could be directly calculated. For this example, the MSE criterion, bias, and standard deviation of the filter (5) as a function of increasing the window length are presented in Fig. 1. The minimum MSE is for $n=5$, which means that the minimum window length should be 6 . For the minimum MSE, bias and standard deviation are in balance (Fig. 1).

\section{B. Steady-State Accuracy}

When the rate of change of frequency drops, a larger window size should be used to minimize the stochastic error that dominates MSE. The effect of measurement noise in the input voltage signals on the estimation accuracy is completely predictable and does not depend on the frequency value. The ratio between standard deviations of the stochastic errors in the signal $\varphi_{m}$ and 


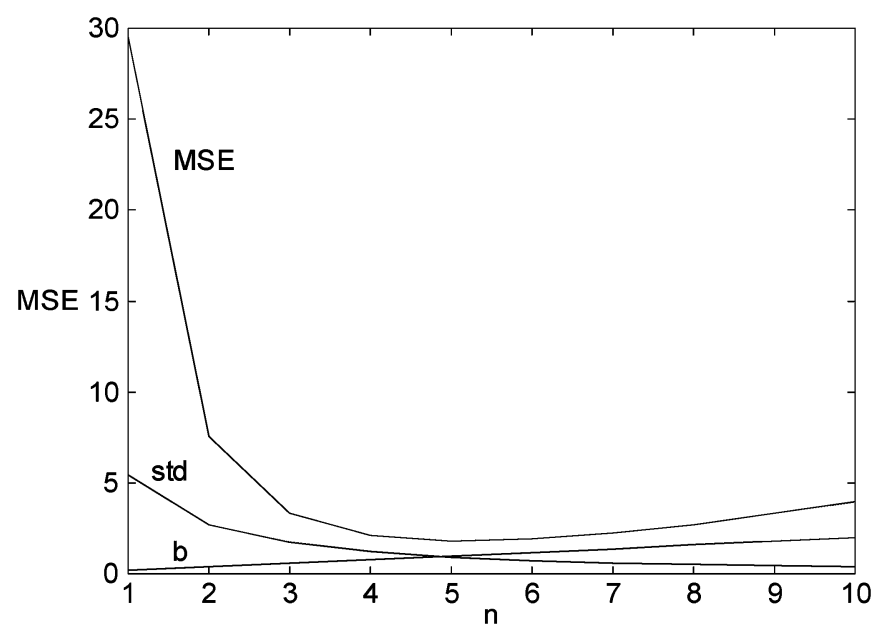

Fig. 1. Mean square error (MSE), bias (b) and standard deviation (std) of the filter (5) as a function of window length (n) when $f^{\prime}=40 \mathrm{~Hz} / \mathrm{s}$ and $\sigma_{e}=0.6 \%$.

TABLE I

RATIO BETWEEN STANDARD DEVIATION OF THE STOCHASTIC ERROR IN FREQUENCY ESTIMATION AND INPUT NOISE STANDARD DEVIATION AS A FUNCTION OF WINDOW LENGTH

\begin{tabular}{|c|c|c|c|c|c|c|}
\hline $\mathrm{n}+1$ & 6 & 12 & 24 & 48 & 96 & 192 \\
\hline$\sigma_{\Delta \omega} / \sigma_{v}$ & 88.3 & 30.9 & 10.9 & 3.85 & 1.36 & 0.48 \\
\hline
\end{tabular}

input three-phase voltages is equal to $1 / \sqrt{3}$. Based on this fact and (9), the ratio between standard deviation $\sigma_{\Delta \omega}$ and input noise standard deviation $\sigma_{v}$ as a function of $n$ can be estimated using

$$
\sigma_{\Delta \omega} / \sigma_{v}=\frac{f_{s}}{\sqrt{3}} \sqrt{\frac{12}{n(n+1)(n+2)}} .
$$

The ratios (12) for the set of dyadic window lengths $(6,12,24$, $48,96,192)$, and the sampling frequency $f_{s}=640 \mathrm{~Hz}$, are calculated and presented in Table I. The adaptive technique should automatically select the largest available window length if the frequency is steady state or slow varying. In this way, the stochastic error will be reduced to a minimum.

Another effect of using larger window lengths is filtering all unwanted oscillatory components in the instantaneous phaseangle signal (2). The frequency response of the differentiation filter (5) is

$$
\begin{aligned}
H_{d}(\omega)= & -f_{s} \sum_{k=0}^{n} h(k, n) e^{-j \omega k} \\
= & -\frac{6 f_{s}}{n(n+1)(n+2)} \\
& \times \frac{(n+2)\left(e^{j \omega}-e^{-j(n-1) \omega}\right)+n\left(e^{-j n \omega}-e^{j 2 \omega}\right)}{\left(e^{j \omega}-1\right)^{2}} .
\end{aligned}
$$

The differentiation filter (5) is the approximation of the ideal differentiator. An ideal differentiator has a frequency response that is linearly proportional to frequency (i.e., $j \omega$ ) (magnitude $\omega$ and phase response $90^{\circ}$ ). The frequency response of the ideal differentiator and differentiator (5) is presented in Fig. 2.
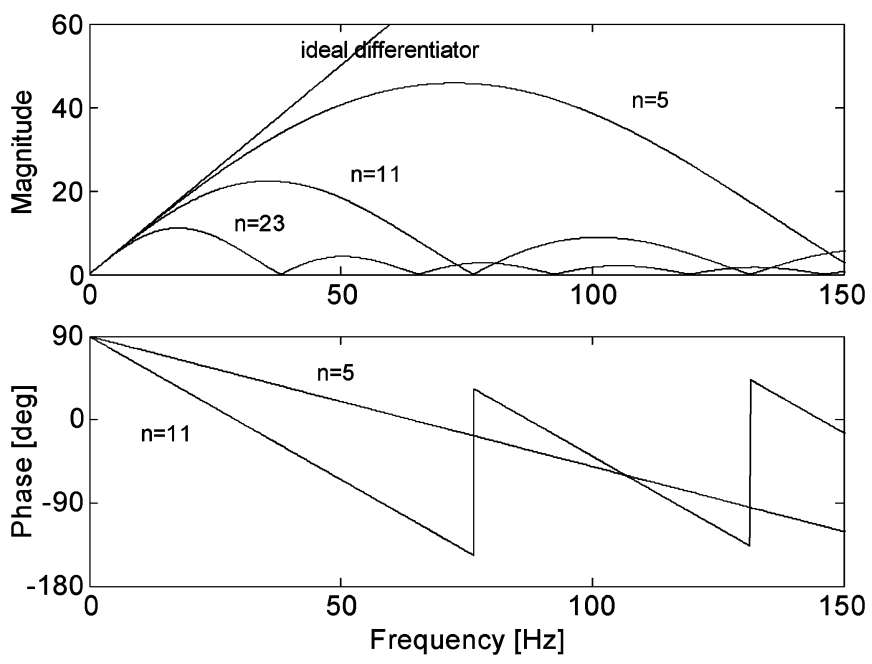

Fig. 2. Magnitude and phase response of the differentiation filter (5) and ideal differentiation filter.

It is clear by observing the magnitude of the frequency response (13) (Fig. 2) that by making the window length small $(n=5)$, we increase the cutoff frequency of the differentiation filter (5). In this case, more noise will pass, but at the same time, more frequencies will be preserved in the differentiated signal. This mode of operation is appropriate when we wish to reduce bias in tracking the fast change of frequency. For steady-state or slow change, the window can be enlarged $(n=11,23$ in Fig. 2) to reduce the cutoff frequency and to have more efficient filtering of noise and oscillatory components in $\varphi_{m}$.

For example, if $10 \mathrm{~Hz}$ is the lowest unwanted frequency in $\varphi_{m}$, the filter (5) with the window of 96 samples $(n=95)$ will have a sufficiently low cutoff frequency.

\section{Window SELECTION AlgORITHM}

If the frequency changes slowly, a large window should be used to filter out noise and oscillatory components, and if the changes are fast, a small window must be used to reduce the bias in frequency tracking. Therefore, a reasonable choice of the window to be used is the one that balances bias and variance as previously discussed.

To find the optimal window length, one needs to calculate estimates (5) for several window lengths. Then, each candidate estimate should be assessed to select the best one. The assessment is based on the intersection of confidence intervals (ICI) method [6], [9]. In this method, after calculating the estimate $\Delta \hat{\omega}(q, n)$ using (5) for a window length $n+1$, the confidence interval is estimated as follows:

$$
\hat{D}(q, n)=\left[\Delta \hat{\omega}(q, n)-\kappa \hat{\sigma}_{\Delta \omega}(n), \Delta \hat{\omega}(q, n)+\kappa \hat{\sigma}_{\Delta \omega}(n)\right]
$$

where $\hat{\sigma}_{\Delta \omega}(n)$ is calculated using the expressions (9) and (10), and $\kappa$ is a threshold of the confidence interval [6].

For each new sample $q$ and each specified window length, the estimates $\Delta \hat{\omega}(q, n)$ and the corresponding confidence intervals $\hat{D}(q, n)$ are calculated. For five window lengths $(6,12,24,48$ and 96), 5 estimates $\Delta \hat{\omega}(q, n)$ and 5 confidence intervals will be calculated for each sample $q$. When the system frequency is in 

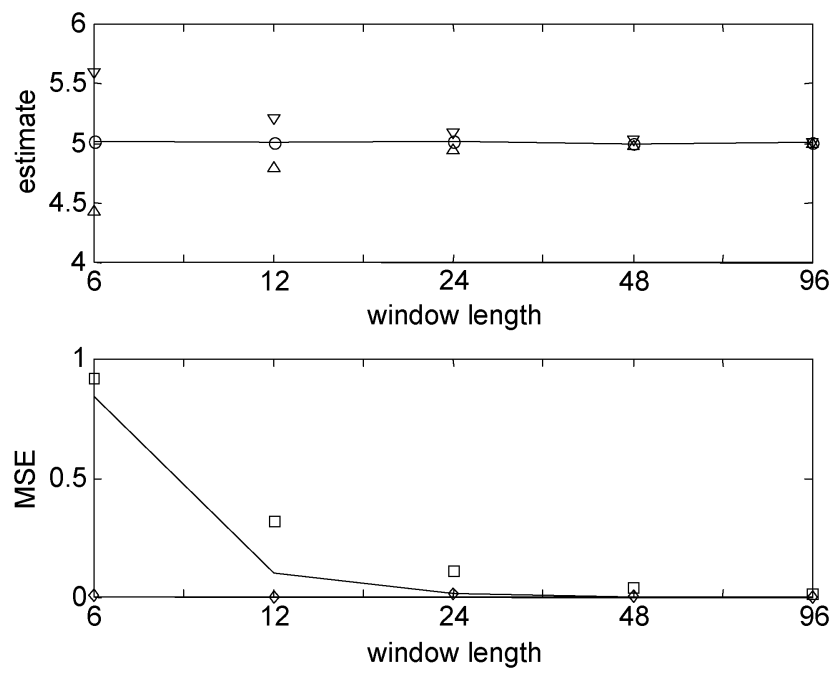

Fig. 3. Steady-state frequency case: Estimates of $\Delta \hat{\omega}(q, n) / 2 \pi$ (circles), common points of confidence intervals $\hat{D}(q, n) / 2 \pi$ (triangles), standard deviations $\hat{\sigma}_{\Delta \omega}(n)$ (square), bias $\hat{b}_{\Delta \omega}(q, n)$ (diamond), and MSE $\hat{J}_{\Delta \omega}(q, n)$ (line) as a function of window length for a time point $q T_{\mathrm{s}}$.
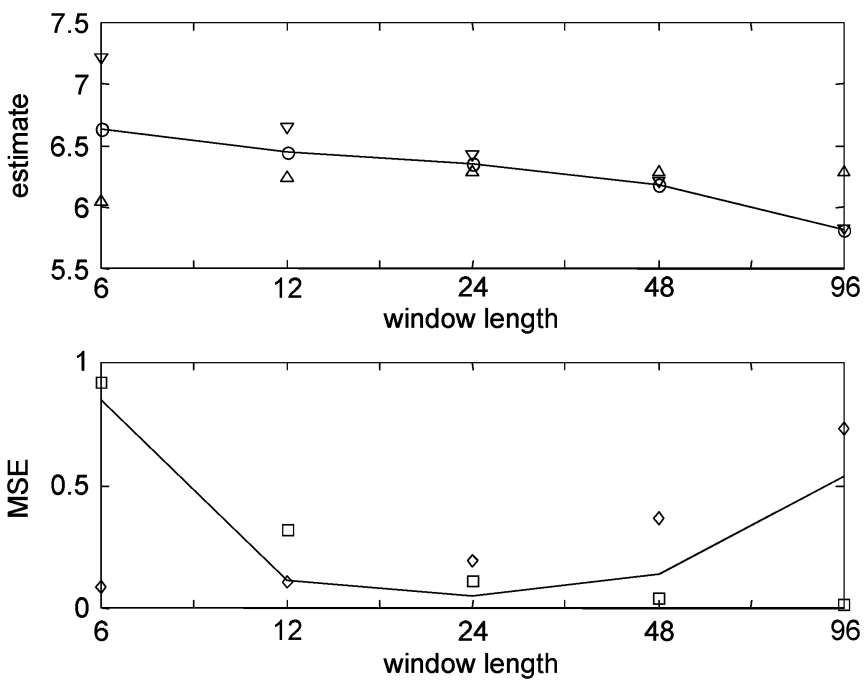

Fig. 4. Ramping frequency case $(10 \mathrm{~Hz} / \mathrm{s})$ : Estimates of $\Delta \hat{\omega}(q, n) / 2 \pi$ (circles), common points of confidence intervals $\hat{D}(q, n) / 2 \pi$ (triangles), standard deviations $\hat{\sigma}_{\Delta \omega}(n)$ (square), bias $\hat{b}_{\Delta \omega}(q, n)$ (diamond), and MSE $\hat{J}_{\Delta \omega}(q, n)$ (line) as a function of window length for a time point $q T_{\mathrm{s}}$.

steady state, the MSE (11) is dominated by the stochastic error in $\varphi_{m}$. By increasing the window length, the standard deviation of this error and the MSE will be reduced, as shown in Fig. 3. The largest window (96 samples) provides the most accurate result. Since the frequency is steady state, the bias remains at zero for all window lengths. In this example, the system frequency is 55 $\mathrm{Hz}$ and the phase-angle measurement error $\sigma_{\varepsilon}=0.6 \%$.

When the system frequency is ramping (for example, $10 \mathrm{~Hz} / \mathrm{s}$ ), the MSE (11) is not only influenced by the stochastic error. As shown in Fig. 4, the MSE is dominated by the stochastic error for small windows and by bias for large windows. The optimal window length ( 24 samples) is clearly the one where the bias and stochastic error are in balance. According to the upper part of Fig. 4, there are common points of the confidence intervals for this window length (24) and previous lengths (6 and 12). The
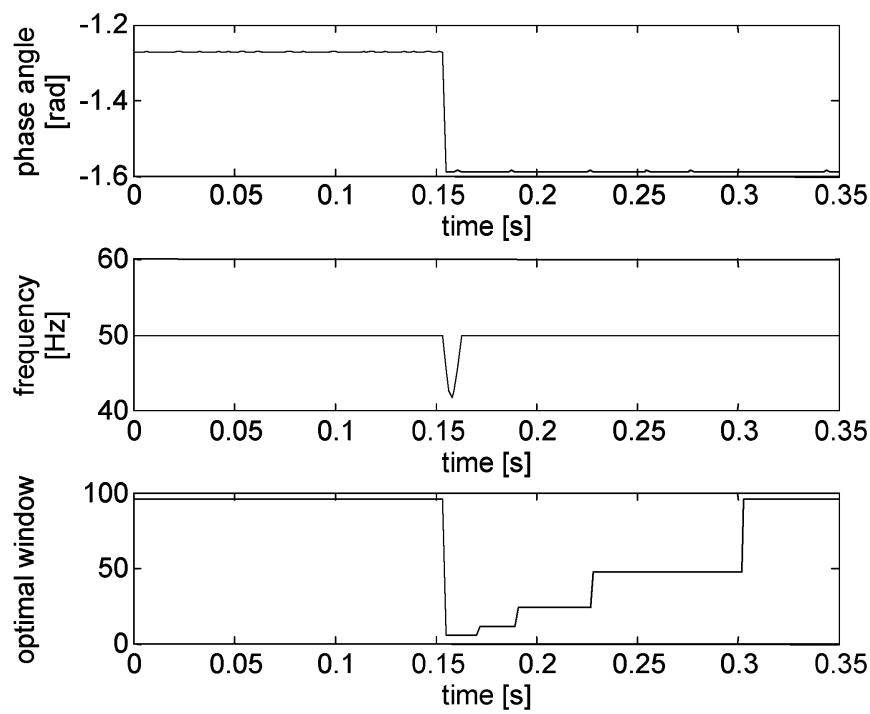

Fig. 5. Response of the window selection algorithm to the phase angle (2) step change.

confidence interval for the estimate based on 48-sample window does not have common points with the previous confidence intervals (window lengths 6,12 , and 24).

The ICI method that determines the optimal point without the need for bias estimate can be summarized as follows: The optimal window length $n^{*}$ for a time instant $q T_{s}$ is the one for which the set $\cap_{n \leq n^{*}} \hat{D}(q, n)$ is nonempty. The asymptotic properties of the ICI method are provided in [9].

The adaptive differentiation filter can be built as a number of parallel filters, which are different in their window size, and the ICI selector, which determines the best size and the corresponding estimate. Another possible fast implementation is based on the frequency-domain solution of the time-domain convolution (5) for a set of dyadic window lengths using the fast Fourier transform (FFT).

\section{Simulation Results AND Discussion}

\section{A. Step Change}

Step changes in magnitude and phase angle of the input threephase voltages can occur in a power system due to faults and switching conditions. Since the proposed technique estimates the frequency through the approximation of the instantaneous phase angle (2), step changes of the angle will cause impulsive frequency estimates. Step changes of magnitude will not have any effect on frequency estimation. The response of the frequency estimator to the phase angle (2) step change is illustrated in Fig. 5. The window selection algorithm handles the angle step change as a large bias and immediately reduces the window to a minimum length. With the smallest window, the frequency estimate returns quickly back to the original value. The impulsive frequency behavior is not realistic and estimation should be blocked in such cases. Immediate step down of the optimal window length to its minimum is the reliable indicator of the phase-angle step change. Return of the frequency estimate to its real value will slow down if the window length is not reduced during the angle step change. Keeping the large 

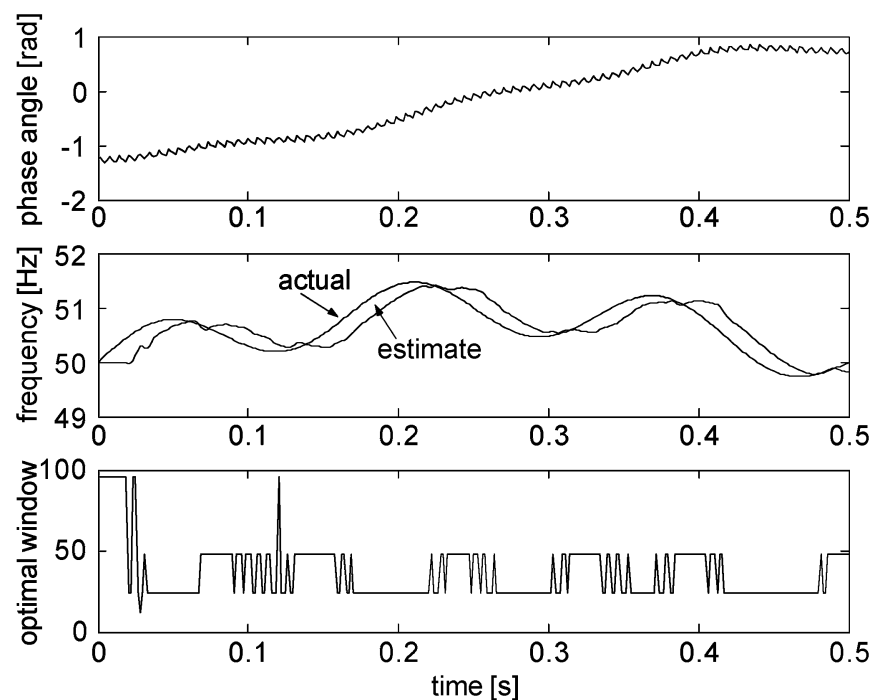

Fig. 6. Tracking of modulated frequency in the presence of harmonics and noise.

window length optimized for filtering noise and harmonics will degrade the tracking performance of the filter.

In this example, the sampling frequency is $640 \mathrm{~Hz}$. The dyadic window lengths $6,12,24,48$, and 96 are selected in order to apply the computationally efficient FFT algorithm [10] for solving the convolution problem (5).

\section{B. Modulated Frequency}

In this simulation example, the nominal frequency is modulated with $1-\mathrm{Hz}$ and $6-\mathrm{Hz}$ signals representing electromechanical transient oscillations. The simulated voltage signals are distorted with normally distributed noise (zero mean and $1 \%$ standard deviation), plus 5\% 3rd harmonic and $2 \% 5$ th harmonic. The sampling frequency in the simulation and in the frequency estimation is $640 \mathrm{~Hz}$. The window lengths are 6, 12, 24, 48, and 96 samples as before. The results are presented in Fig. 6 .

The third block in Fig. 6 illustrates the process of selecting the optimal window to adapt the filter to the underlying frequency. When the instantaneous frequency is steady-state or it is changing slowly, the window is enlarged to 48 samples making the differentiation filter more efficient in filtering noise and harmonics. For faster changing instantaneous frequency, the window length drops to 24 . This will reduce bias but the filtering efficiency is degraded. The result could be improved by using a denser set of window lengths. Another improvement would be the use of a better approximation of the instantaneous phase angle in (4) applying second- or third-order polynomial [11]. In the offline applications, the estimates obtained with left, central, and right windows could be combined to produce very accurate results [12].

\section{APPLICATION EXAMPLES}

\section{A. Resonant Frequency}

The frequency-tracking algorithm has been tested using the voltage signals recorded during a single-phase-to-ground shortcircuit (white phase) at a $765-\mathrm{kV}$ line in South Africa. This line is $434 \mathrm{~km}$ long and it is equipped with shunt reactors. White
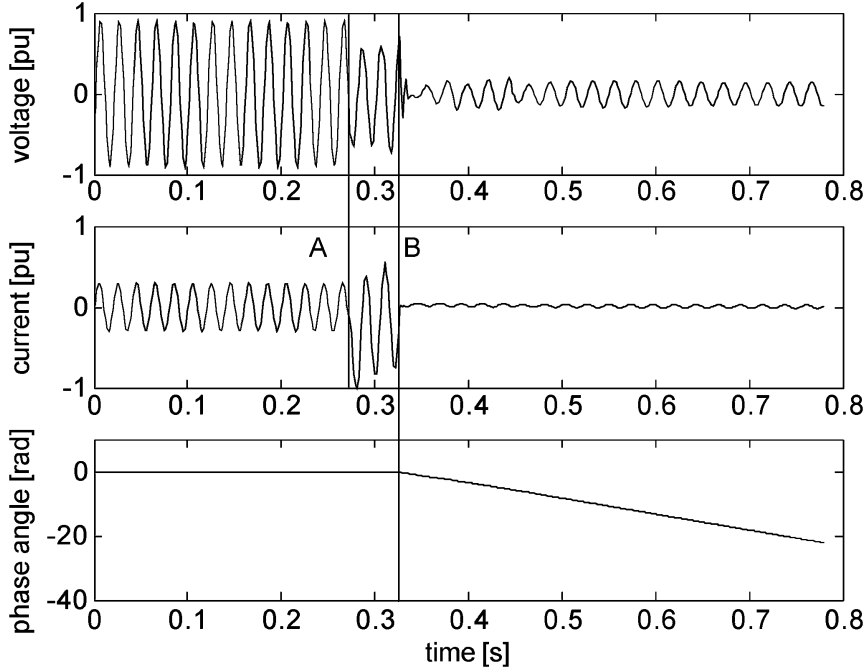

Fig. 7. White phase voltage and current signals (normalized) recorded during fault condition on a $765-\mathrm{kV}$ line, and the instantaneous phase angle. Sequence of events: A-fault inception, B-breakers open.
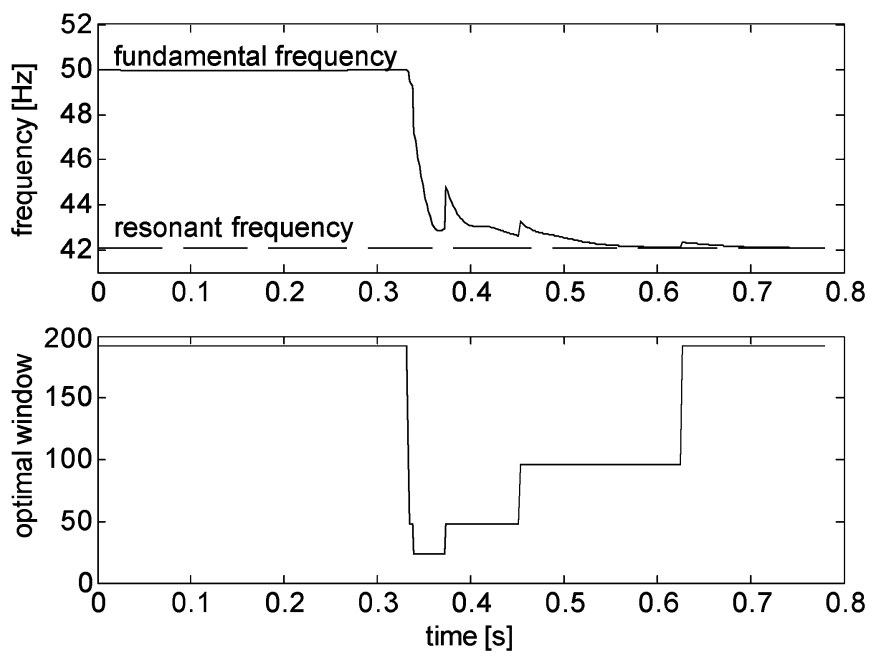

Fig. 8. Frequency tracking on a $765-\mathrm{kV}$ line with a shunt reactor during fault conditions.

phase voltage and current signals are shown in Fig. 7 together with the estimated instantaneous phase angle $\varphi_{m}$. The sampling frequency is $640 \mathrm{~Hz}$. The window lengths used by the algorithm are 6, 12, 24, 48, 96, and 192.

Position A in Fig. 7 indicates the fault inception. Position B is the moment when the fault has been isolated. Due to accumulated energy in the circuit, the voltage does not disappear. An oscillating voltage waveform can be seen in Fig. 7, which slowly reduces in magnitude. This oscillation is a result of the interaction between the reactors and the capacitance of the line. This is a parallel resonant circuit. At B in Fig. 7, the instantaneous phase angle $\varphi_{m}$ suddenly started to change because the frequency now differs from the fundamental. The result of the adaptive frequency-tracking algorithm is shown in Fig. 8. The algorithm automatically reduces the window length to change quickly from the fundamental to resonant frequency. After the transition, the window length is enlarged again to reduce noise and achieve maximum accuracy. 

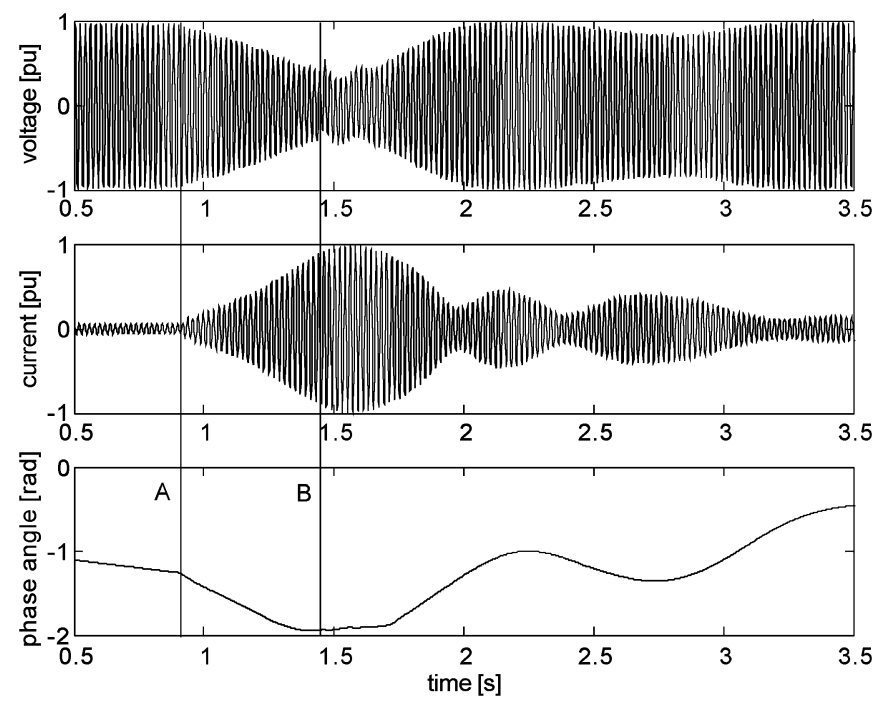

Fig. 9. Red phase voltage and current signals (normalized) recorded during power swing condition on a $400-\mathrm{kV}$ line, and the instantaneous phase angle. Sequence of events: A-power swing inception, B-CVT transients inception.

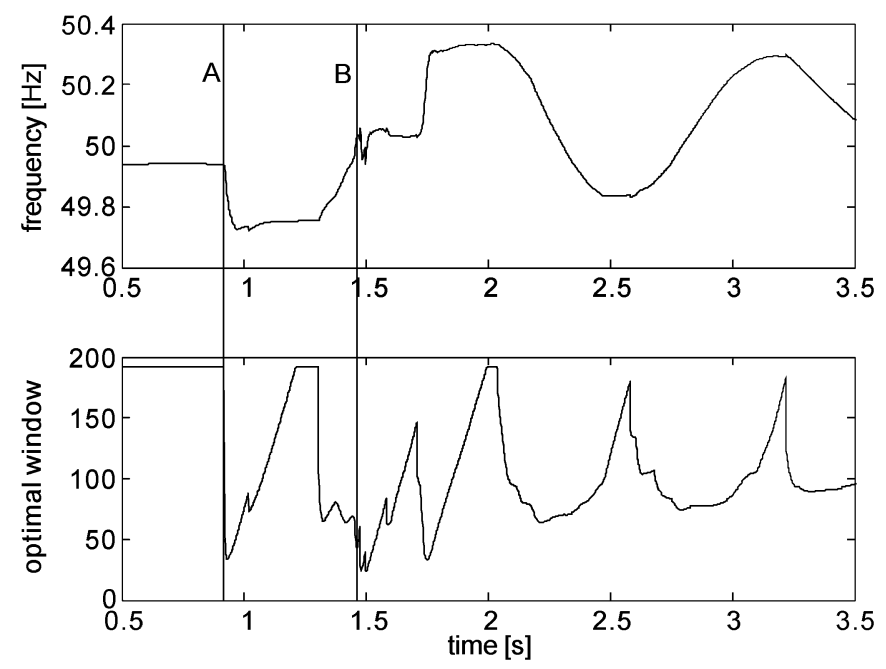

Fig. 10. Frequency tracking on a $400-\mathrm{kV}$ line during the power swing condition.

\section{B. Power Swing}

The transmission network in South Africa is structured in such a manner that a major load center is situated at distance of $1500 \mathrm{~km}$ from the pool of power stations. The link to the pool of power plants goes through a number of $400-\mathrm{kV}$ lines with series capacitor compensation. The loss of one of these tie-lines resulted in a severe power swing condition shown in Fig. 9. Fig. 9 presents the red phase voltage and current signals as well as the instantaneous phase angle $\varphi_{m}$. Location A indicates the inception of a power swing condition. The start of the capacitive voltage transformer (CVT) transients is designated with B in Fig. 9. The sampling frequency is $640 \mathrm{~Hz}$. The set of all window lengths from 6 till 192 samples is used in this application.

The obtained frequency estimate using the adaptive algorithm is shown in Fig. 10. The adaptation of the window length to instantaneous frequency changes is presented in the same figure. This illustrates how the algorithm automatically uses large window lengths for slow-varying or steady-state instantaneous frequency and small window lengths for fast changes. It should be noticed that a linear function has been used to model the instantaneous phase angle on the left-sided window of a variable length. For example, the largest window length has been selected for the estimate at $2 \mathrm{~s}$ (Fig. 10) because samples in such a long window can be accurately approximated with a linear function as can be observed in Fig. 9. At approximately $2.25 \mathrm{~s}$ (Fig. 9), the instantaneous phase angle is at the peak and a linear function is a good approximation only for a smaller number of samples. As shown in Fig. 10, the window length drops from 192 to 70 samples. The cyclic change of window length in Fig. 10 follows the change of the instantaneous phase angle.

At the CVT transients inception moment (location B), the use of very small window lengths resulted in a very fast return to accurate frequency tracking. The effect of the instantaneous change in the voltage phase angle during this condition is quickly eliminated.

\section{CONCLUSION}

This paper describes the adaptive technique for frequency measurement, where the window size is varied based on the frequency deviation from the nominal frequency. The technique is based on the optimal local approximation of the instantaneous voltage phase angle. One of the problems of frequency estimation algorithms using phase angles is the erroneous frequency estimates when a sudden change in phase angle is seen during switching conditions. This problem is efficiently handled by dynamically reducing the window length for those conditions there by eliminating the bad samples quickly. The application and simulation examples show that the technique is very robust and accurate in measuring the instantaneous frequency under various practical conditions.

\section{APPENDIX}

The bias of the filter (5) is defined as

$$
\hat{b}_{\Delta \omega}(q, n)=E[\Delta \hat{\omega}(q, n)]-\Delta \omega(q)
$$

where $E[*]$ is the expected value. By using the filter expression (5) in (15), we obtained

$$
\hat{b}_{\Delta \omega}(q, n)=-\frac{1}{T_{s}} \sum_{k=0}^{n} h(k, n) E\left[\varphi_{m}(q-k)\right]-\Delta \omega(q) .
$$

The phase angle $\varphi_{m}$ in (16) can be modeled using a nonlinear function plus modeling error $\varepsilon$. It is assumed that the error $\varepsilon$ belongs to the class of independently and identically distributed random numbers with zero mean and the variance $\sigma_{\varepsilon}^{2}$. The nonlinear function is approximated using the second-order Taylor's series. Hence, the model for the phase angle is

$\varphi_{m}(q-k)=\varphi(q)-\Delta \omega(q) k T_{s}+\Delta \omega^{\prime}(q) \frac{\left(k T_{s}\right)^{2}}{2}+\varepsilon(q-k)$ 
Incorporating this model in (16), we have

$$
\begin{array}{r}
\hat{b}_{\Delta \omega}(q, n)=-\frac{1}{T_{s}} \sum_{k=0}^{n} h(k, n) \varphi(q)+\sum_{k=0}^{n} k h(k, n) \Delta \omega(q) \\
-\frac{T_{s}}{2} \sum_{k=0}^{n} k^{2} h(k, n) \Delta \omega^{\prime}(q)-\Delta \omega(q) .
\end{array}
$$

The following properties of the weight sequence (6):

$$
\begin{aligned}
\sum_{k=0}^{n} h(k, n) & =\sum_{k=0}^{n} \frac{6(2 k-n)}{n(n+1)(n+2)}=0, \\
\sum_{k=0}^{n} k h(k, n) & =\sum_{k=0}^{n} k \frac{6(2 k-n)}{n(n+1)(n+2)}=1, \\
\sum_{k=0}^{n} k^{2} h(k, n) & =\sum_{k=0}^{n} k^{2} \frac{6(2 k-n)}{n(n+1)(n+2)}=n
\end{aligned}
$$

are used in (18) to obtain the bias expression (8).

The variance of the filter (5) is defined as

$$
\hat{\sigma}_{\Delta \omega}^{2}(n)=E\left\{(\Delta \hat{\omega}(q, n)-E[\Delta \hat{\omega}(q, n)])^{2}\right\} .
$$

Replacing $\Delta \hat{\omega}(q, n)$ in (20) with the filter expression (5) and approximating $\varphi_{m}$ with the model (17), we obtained

$$
\hat{\sigma}_{\Delta \omega}^{2}(n)=\frac{1}{T_{s}^{2}} \sum_{k=0}^{n} h(k, n)^{2} \sigma_{\varepsilon}^{2}
$$

The following property of the weight sequence (6):

$$
\sum_{k=0}^{n}\left(\frac{6(2 k-n)}{n(n+1)(n+2)}\right)^{2}=\frac{12}{n(n+1)(n+2)}
$$

is used in (21) to obtain the standard deviation expression (9).

\section{REFERENCES}

[1] IEEE Tutorial Course, Advancements in microprocessor based protection and communication pp. 8-25, 1997.

[2] E. Palmer and J. De La Ree, "A new method for computing instantaneous voltage angles," in Proc. Fault and Disturbance Analysis Precise Measurements in Power Systems Conf., Arlington, VA, 1995.

[3] M. Akke, "Frequency estimation by demodulation of two complex signals," IEEE Trans. Power Del., vol. 12, no. 1, pp. 157-163, Jan. 1997.

[4] R. W. Hamming, Digital Filters. Englewood Cliffs, NJ: Prentice-Hall, 1983, pp. 118-126.

[5] E. Clarke, Circuit Analysis of AC Power Systems. New York: Wiley, 1950, vol. 1.

[6] V. Katkovnik, "A new method for varying adaptive bandwidth selection," IEEE Trans. Signal Process., vol. 47, no. 9, pp. 2567-2571, Sep. 1999.

[7] W. N. Venables and B. D. Ripley, Modern Applied Statistics With S-Plus. New York: Springer-Verlag, 1999, pp. 126-132.

[8] J. Fan and I. Gijbels, Local Polynomial Modelling and Its Application. London, U.K.: Chapman \& Hall, 1996.

[9] A. Goldenshluger and A. Nemirovski, "On spatial adaptive estimation of nonparametric regression," Math. Methods Stat., vol. 6, no. 2, pp. 135-170, 1997.

[10] J. G. Proakis and D. G. Manolakis, Digital Signal Processing: Principles, Algorithms, and Applications. Englewood Cliffs, NJ: Prentice-Hall, 1996, ch. 6.

[11] R. Živanović, "Nonparametric frequency estimation for power system applications," presented at the IEEE Porto Power Tech, Porto, Portugal, Sep. 2001, paper no. 0-7803-7139-9/01, Session QSR1-069.

[12] _ "A nonparametric technique for high precision estimation of power system time-varying phasors," presented at the 14th Power Systems Comput. Conf., Seville, Spain, Jun. 2002, paper 1, Session 37.

Rastko Živanović (M'97) received the Dipl.Ing. and M.Sc. degrees from the University of Belgrade, Belgrade, Serbia, in 1987 and 1991, respectively, and the Ph.D. degree from the University of Cape Town, Cape Town, South Africa, in 1997.

Currently, he is with the School of Electrical and Electronic Engineering, the University of Adelaide, Adelaide, Australia. His research interests include power system protection and control. 